\section{The opposing effects of acute inflammation and iron deficiency anemia on serum hepcidin and iron absorption in young women}

\author{
Nicole U. Stoffel, ${ }^{1}$ Meryem Lazrak, ${ }^{2}$ Souhaila Bellitir, ${ }^{2}$ Nissrine El Mir, ${ }^{2}$ \\ Asmaa El Hamdouchi, ${ }^{2}$ Amina Barkat, ${ }^{3}$ Christophe Zeder, ${ }^{1}$ Diego Moretti, ${ }^{1}$ \\ Hassan Aguenaou ${ }^{2}$ and Michael B. Zimmermann ${ }^{1}$
}

${ }^{1}$ ETH Zürich, Laboratory of Human Nutrition, Institute of Food Nutrition and Health, Department of Health Science and Technology, Zürich, Switzerland; ' ${ }^{I}$ bn Tofaïl UniversityCNESTEN, Joint Research Unit in Nutrition and Food, RDC-Nutrition AFRA/IAEA, RabatKénitra, Morocco and ${ }^{3}$ Mohamed V University, Unit of Research on Nutrition and Health of Mother and Nutrition, Faculty of Medicine and Pharmacy, Rabat, Morocco

\section{ABSTRACT}

$\mathrm{H}$ patic hepcidin synthesis is stimulated by inflammation but inhibited during iron deficiency anemia (IDA). In humans, the relative strength of these opposing signals on serum hepcidin and the net effect on iron absorption and systemic iron recycling is uncertain. In this prospective, 45 -day study, in young women ( $n=46$; age $18-49$ years) with or without IDA, we compared iron and inflammation markers, serum hepcidin and erythrocyte iron incorporation from ${ }^{57} \mathrm{Fe}$-labeled test meals, before and 8, 24 and 36 hours (h) after influenza/DPT vaccination as an acute inflammatory stimulus. Compared to baseline, at $24-36 \mathrm{~h}$ after vaccination: 1 ) interleukin-6 increased 2-3-fold in both groups $(P<0.001)$; 2$)$ serum hepcidin increased $>2$-fold in the non-anemic group $(P<0.001)$, but did not significantly change in the IDA group; 3$)$ serum iron decreased in the non-anemic group $(P<0.05)$ but did not change in the IDA group; and 4$)$ erythrocyte iron incorporation did not change in either of the two groups, but was approximately 2 -fold higher in the IDA group both before and after vaccination $(P<0.001)$. In this study, mild acute inflammation did not increase serum hepcidin in women with IDA, suggesting low iron status and erythropoietic drive offset the inflammatory stimulus on hepcidin expression. In non-anemic women, inflammation increased serum hepcidin and produced mild hypoferremia, but did not reduce dietary iron absorption, suggesting iron-recycling macrophages are more sensitive than the enterocyte to high serum hepcidin during inflammation. The study was registered as a prospective observational trial at clinicaltrials.gov identifier: 02175888. The study was funded by the International Atomic Energy Agency.

\section{Introduction}

The hepatic hormone hepcidin regulates serum iron concentrations and iron homeostasis. ${ }^{1}$ Hepcidin synthesis is stimulated by high liver iron stores, high plasma iron concentrations and inflammation, and is inhibited by hypoxia and erythropoietic drive. ${ }^{1}$ In conditions such as the iron-loading anemias, the anemia of chronic disease, and acute infection during iron deficiency anemia (IDA), several of these factors simultaneously generate opposing signals on hepcidin expression. The relative strength of these opposing signals to regulate serum hepcidin (SHep) in humans is uncertain.

Iron-deficient mice up-regulate hepcidin expression when injected with lipopolysaccharide (LPS) to induce inflammation. ${ }^{2}$ Other animal studies suggest erythroid demand for iron is a stronger regulator of hepcidin expression than inflammation..$^{3.5}$ Most human studies examining opposing stimuli on SHep have been descriptive; some have suggested that iron status and/or erythropoiesis are the major regulators of SHep, ${ }^{4,6,7}$ others suggest inflammation is associated with
Haematologica 2019

Volume 104(6):1143-1149

\section{Correspondence:}

NICOLE U. STOFFEL

nicole.stoffe!@hest.ethz.ch

Received: October 8, 2018.

Accepted: January 2, 2019.

Pre-published: January 10, 2019.

doi:10.3324/haematol.2018.208645

Check the online version for the most updated information on this article, online supplements, and information on authorship \& disclosures: www.haematologica.org/content/104/6/1143

(C)2019 Ferrata Storti Foundation

Material published in Haematologica is covered by copyright. All rights are reserved to the Ferrata Storti Foundation. Use of published material is allowed under the following terms and conditions:

https://creativecommons.org/licenses/by-nc/4.0/legalcode. Copies of published material are allowed for personal or internal use. Sharing published material for non-commercial purposes is subject to the following conditions:

https://creativecommons.org/licenses/by-nc/4.0/legalcode, sect. 3. Reproducing and sharing published material for commercial purposes is not allowed without permission in writing from the publisher. 
high SHep even in anemic subjects. ${ }^{8,9}$ Nearly all experimental data are derived from cells or mice, where often very strong stimuli are applied (e.g. injection of LPS, $2,3,5$ phlebotomy, ${ }^{4}$ severe iron deficiency $\left.{ }^{2,5}\right)$. In humans, there is a lack of experimental data describing the effect of milder, physiological changes in these opposing stimuli on SHep and the net effects on iron recycling and iron absorption, but these interactions are common and relevant in many disorders.

Therefore, the objective of this prospective study was to assess changes in iron markers, SHep, iron absorption and erythrocyte iron incorporation during acute inflammation, in both non-anemic women and women with IDA. Previous human studies reported the effects of an acute inflammatory stimulus [e.g. infusions of LPS $^{10}$ or interleukin-6 (IL-6) ${ }^{11}$ ] on SHep, but they did not assess effects on iron absorption, or how anemia or iron status may modulate this response. As the inflammatory stimulus in this study, we used vaccination, a practical, safe and standardized model for the study of mild-to-moderate inflammation in humans. ${ }^{12,13}$ Our hypotheses were: 1) in nonanemic women, vaccination would induce acute inflammation and increase Shep. This would decrease iron absorption and produce hypoferremia; and 2) in contrast, in women with IDA, vaccination would induce acute inflammation, but would not increase SHep or affect serum iron or iron absorption. We used stable iron isotope techniques to quantify erythrocyte iron incorporation of dietary iron before and after vaccination.

\section{Methods}

\section{Study subjects}

We recruited women from the staff of the University Hospital Ibn Sina in Rabat, Morocco. Detailed inclusion criteria are described in the Online Supplementary Appendix. In this prospective, 45-day study, in women ( $n=46$, age $18-49$ years) with IDA or without anemia, we compared iron and inflammation markers and SHep before and 8, 24 and 36 hours (h) after influenza/diphtheria-tetanus-pertussis (DTP) vaccination and erythrocyte iron incorporation from ${ }^{57}$ Fe-labeled test meals, before and $24 \mathrm{~h}$ after the vaccination as an acute inflammatory stimulus (Figure 1). The study was approved by the ethics committees of the ETH Zurich, Zurich, Switzerland and the University Mohammed V, Rabat, Morocco. All participants gave informed written consent.

On study day 1, an afternoon baseline blood sample was taken. On study day 2, after an overnight fast, a baseline morning blood sample was taken and we administered a test meal containing 6 mg labeled ${ }^{57} \mathrm{Fe}$ as ethylenediaminetetraacetic acid ferric sodium salt (NaFeEDTA), added to a standardized test meal, given with bottled water, as described in the Online Supplementary Appendix. Blood samples were taken in the afternoon on day 2 as well as the next morning (day 3). After a 19-day isotope incorporation period, on study day 22 , a blood sample was taken to measure erythrocyte iron incorporation; this blood sample also served as the new baseline afternoon sample for the second absorption study. In the morning of day 23, a morning blood sample was taken. Then, all subjects received the trivalent Influenza Virus Vaccine Vaxigrip (Sanofi Pasteur, Lyon, France) and the DTP Virus Vaccine Dultavax (Sanofi Pasteur) given intramuscularly. Blood samples were taken at $8 \mathrm{~h}, 24 \mathrm{~h}$ and $36 \mathrm{~h}$ after vaccination. At $24 \mathrm{~h}$ after vaccination, on study day 24 , an identical labeled test meal was administered, as described above. The final blood sample was taken on day 45 . We assessed total and fractional iron absorption (FIA) by measur- ing the amount of stable isotopic tracers incorporated in red blood cells 19 days after administration of the labeled test meals. ${ }^{14-16}$ Hemoglobin $(\mathrm{Hb})$, iron- and inflammatory biomarkers were measured as described in the Online Supplementary Appendix.

Assuming a standard deviation (SD) of 0.20 on differences in log transformed erythrocyte iron incorporation from previous ETH studies, a type I error rate of $5 \%$ and $80 \%$ power, we expected to detect a difference in FIA of $35 \%$ within groups with a sample size of 20 subjects per group. Assuming a drop-out rate of $20 \%$, we enrolled 50 women ( 25 anemic and 25 non-anemic women).

\section{Statistical analysis}

We performed the statistical analyses using SPSS (IBM SPSS statistics, v.22), as described in detail in the Online Supplementary Appendix. We used linear mixed effect model analysis to assess the effect of the group (anemic vs. non-anemic) and treatment (vaccination) on different variables. Group and treatment were defined as fixed effects, participants as random intercept effects using a variance component structure matrix. Regression analyses were performed with SHep, FIA and serum iron as dependent variables. Pearson and Spearman correlations were applied. For withingroup effects, dependent sample $t$-tests or related samples nonparametric tests were used. $P<0.05$ was considered significant.

\section{Results}

We began recruiting on $1^{\text {st }}$ September 2017, and from September 2017 to February 2018 we enrolled 50 women (28 non-anemic and 22 with IDA) into the study. We completed the study on $29^{\text {th }}$ March 2018. Six women in the non-anemic group left the study because they no longer wanted to participate in the study: three before the 19 day blood sample (when we measured erythrocyte iron incorporation from the first test meal) and three after the 19 day blood sample. In the IDA group, one subject left the study before the 19 day blood sample because she no longer wanted to participate in the study (Figure 1). Data from the three non-anemic women who left the study after the 19 day blood sample were included in the analytical models, resulting in a total of $n=46$ women ( 21 with IDA and 25 non-anemic).

Baseline characteristics of the subjects by group are shown in Table 1. There were no significant betweengroup differences in age, Body Mass Index or markers of inflammation, and no subject had increased markers of inflammation. There were significant between-group differences in hemoglobin ( $\mathrm{Hb})$, serum ferritin (SF), soluble transferrin receptor (sTfR), body iron stores (BIS), Shep, and erythropoietin (EPO) (all $P<0.001$ ). Eight of the women in the non-anemic group were iron-deficient, as defined by $\mathrm{SF}<15 \mu \mathrm{g} / \mathrm{L}^{17}$

There was a significant vaccination effect $(P<0.001)$ on IL-6, but no significant group effect or group-vaccination interaction (Table 2). There were no significant betweengroup differences in IL- 6 measured at 4:00 pm the day before and at 8:00 am just before vaccination or at 8 h, 24 $\mathrm{h}$, and $36 \mathrm{~h}$ after vaccination (Figure 2A). Median interquartile range (IOR) IL-6 (pg/mL) significantly increased in both groups comparing baseline to $24 \mathrm{~h}$ after vaccination $(P<0.001)$ : in the non-anemic group from 1.12 $(0.92-1.66)$ to 3.37 (2.88-4.32), and in the IDA group from 1.53 (1.41-1.81) to 3.14 (2.48-4.33) (Table 2).

There was a significant group $(P<0.001)$ and vaccination $(P<0.001)$ effect on SHep, but no significant group-vacci- 
nation interaction (Table 2). There were significant between-group differences in SHep measured at 4:00 pm and at 8:00 am before vaccination and at $8 \mathrm{~h}, 24 \mathrm{~h}$, and 36 $\mathrm{h}$ after vaccination (all $\mathrm{P}<0.05$ ) (Figure $2 \mathrm{~B}$ ). In the non-anemic group, median IOR SHep (nM) significantly increased from $1.60(0.93-2.86)$ before to $3.56(1.04-5.53)$ at $24 \mathrm{~h}$ after vaccination $(P<0.001)$. In contrast, in the IDA group, vaccination did not induce a significant increase in SHep: median (IOR) SHep (nM) was 0.45 (0.23-0.61) before and 0.45 (0.32-1.17) $24 \mathrm{~h}$ after vaccination (Table 2).

There was a significant group effect $(P<0.001)$ and group-vaccination interaction $(P<0.001)$ on serum iron and transferrin saturation (TSAT), but no significant vaccination effect (Table 3). There were significant betweengroup differences in serum iron and TSAT at baseline $(P<0.001$ for both $)$ and at $24 \mathrm{~h}$ after vaccination $(P<0.05$ for both). In the non-anemic group, geometric mean [-Standard Deviation (SD), +SD] serum iron $(\mu \mathrm{g} / \mathrm{mL})$ significantly decreased from $0.91(0.71,1.17)$ before to 0.78
$(0.59,1.02)$ at $24 \mathrm{~h}$ after vaccination $(P<0.05)$ (Figure $3 \mathrm{~A})$. Geometric mean (-SD, +SD) TSAT (\%) decreased from $19.19(14.69,25.07)$ before to $16.23(12.39,21.26)$ at $24 \mathrm{~h}$ after vaccination $(P=0.066)$. In the IDA group, vaccination had no significant effect on either serum iron or TSAT.

There was a significant group effect on FIA (erythrocyte iron incorporation) $(P<0.001)$, but no significant vaccination effect or group-vaccination interaction (Table 2). Comparing erythrocyte iron incorporation before and after vaccination, there were no significant differences within either of the two groups (Figure 3B). However, between groups, erythrocyte iron incorporation was significantly higher (by approx. 2-fold) in women with IDA both before and after vaccination (Table 2 ).

Iron and inflammation indicators at $24 \mathrm{~h}$ after vaccination are shown in Table 3 . There was a significant group effect on SF, sTfR, BIS, EPO $(P<0.001)$, but none on CRP or AGP. There was a significant vaccination effect on $s$ TfR $(P<0.01)$, CRP $(P<0.05)$ and AGP $(P<0.001)$; all of these

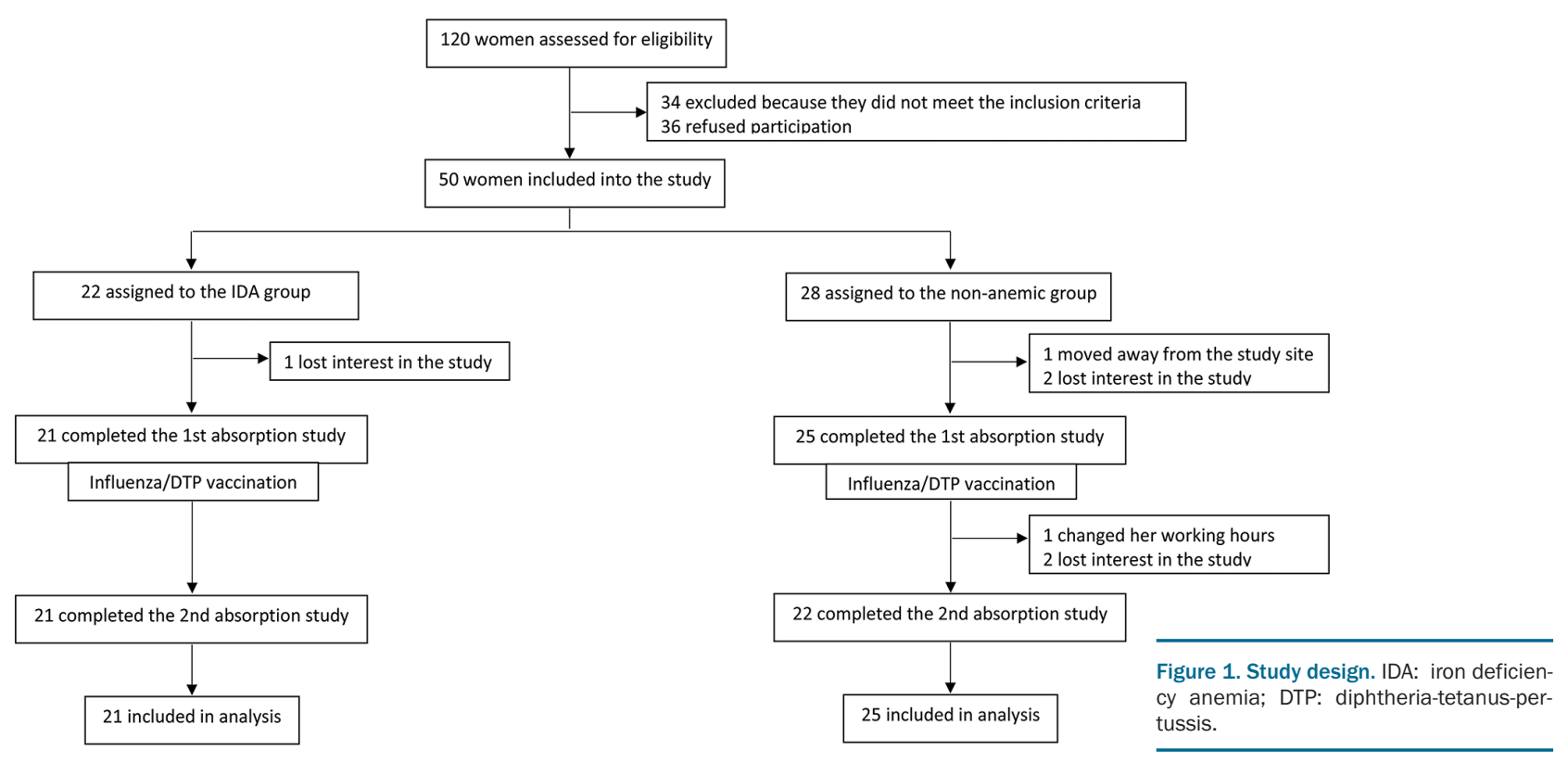

Table 1. Baseline characteristics of the women $(n=46)$ in the iron deficiency anemia group and the non-anemic group.

\begin{tabular}{|c|c|c|}
\hline & IDA (n=21) & Non-anemic $(n=25)$ \\
\hline Age, years* & $23(21-27)$ & $24(23-30)$ \\
\hline Body Mass Index, $\mathrm{kg} / \mathrm{m}^{2 * *}$ & $22.9 \pm 2.5$ & $22.3 \pm 3.1$ \\
\hline Hemoglobin, $g / \mathrm{dL}^{*}$ & $11 \cdot 3(10.7-11.6)^{\mathscr{\Psi}}$ & $13 \cdot 3(12.6-13.9)$ \\
\hline Serum ferritin, $\mu \mathrm{g} / \mathrm{L}^{* * *}$ & $6.3(3.9,10.3)^{\llbracket /}$ & $23.8(10.7,52.9)$ \\
\hline Serum transferrin receptor, $\mathrm{mg} / \mathrm{L}^{* * *}$ & $8.33(6.08,11.43)^{\pi}$ & $5.99(4.60,7.79)$ \\
\hline Body iron stores, mg/kg BW** & $-2.5 \pm 2.7^{\mathbb{I}}$ & $3.5 \pm 3.5$ \\
\hline Serum iron, $\mu \mathrm{g} / \mathrm{mL}^{* * *}$ & $0.51(0.33,0.78)^{\pi}$ & $0.91(0.71,1.17)$ \\
\hline Total iron binding capacity, $\mu \mathrm{g} / \mathrm{mL}^{*}$ & $4.79(4.49-5.06)$ & $4.81(4.41-5.01)$ \\
\hline Transferrin saturation, $\% * * *$ & $10.78(6.89,16.89)^{\mathbb{I}}$ & $19.19(14.69,25.07)$ \\
\hline Erythropoietin, mIU/mL*** & $20.37(11.48,36.17)^{\mathscr{q}}$ & $8.61(5.40,13.74)$ \\
\hline C-reactive protein, $\mathrm{mg} / \mathrm{L}^{* * *}$ & $0.70(0.18,2.76)$ & $0.59(0.13,2.59)$ \\
\hline Alpha-1-acid glycoprotein, $g / L^{* * *}$ & $0.71(0.51,0.98)$ & $0.72(0.49,1.05)$ \\
\hline
\end{tabular}


variables were lower after vaccination, and the decrease in sTfR in the IDA group reached borderline significance $(P=0.063)$. There was a significant group-vaccination interaction on SF $(P<0.001)$ and BIS $(P<0.01)$.

There was no correlation between IL- 6 and SHep at baseline in either group. At $24 \mathrm{~h}$ after vaccination, IL- 6 and SHep significantly correlated in the non-anemic group $(\mathrm{r}=0.426 ; P<0.05)$, but did not in the IDA group. In the regressions including all subjects, sTfR, $\mathrm{Hb}$, EPO and IL-6 explained $55 \%$ and $62 \%$ of the variation of SHep at baseline and at $24 \mathrm{~h}$ after vaccination, respectively. At baseline in all subjects, the only significant predictors of SHep were $\mathrm{EPO}(\beta=-0.570 ; P<0.01)$ and $\mathrm{Hb}(\beta=0.372 ; P<0.05)$. In contrast, at $24 \mathrm{~h}$ after vaccination, the only significant predic-

Table 2. Serum interleukin-6 (IL-6), serum hepcidin and erythrocyte iron incorporation (iron absorption and utilization), at baseline and 24 hours after vaccination, in the iron deficiency anemia group and the non-anemic group.

\begin{tabular}{|c|c|c|c|c|c|}
\hline & $\begin{array}{c}\text { Baseline } \\
(n=46)\end{array}$ & $\begin{array}{l}\text { 24h after vaccination } \\
\qquad(n=43)\end{array}$ & $\begin{array}{l}\text { Group } \\
\text { effect }\end{array}$ & $\begin{array}{c}P \\
\text { Vaccination } \\
\text { effect }\end{array}$ & $\begin{array}{c}\text { Group-vaccination } \\
\text { effect }\end{array}$ \\
\hline $\begin{array}{l}\mathrm{IL}-6, \mathrm{pg} / \mathrm{mL} \\
\text { IDA } \\
\text { Non-anemic }\end{array}$ & $\begin{array}{l}1.53(1.41-1.81) \\
1.12(0.92-1.66)\end{array}$ & $\begin{array}{l}3.14(2.48-4.33)^{\circ} \\
3.37(2.88-4.32)^{\circ}\end{array}$ & 0.212 & $<0.001$ & 0.349 \\
\hline $\begin{array}{l}\text { Serum hepcidin, nM } \\
\text { IDA } \\
\text { Non-anemic }\end{array}$ & $\begin{array}{l}0.45(0.23-0.61)^{*} \\
1.60(0.93-2.86)\end{array}$ & $\begin{array}{l}0.45(0.32-1.17)^{*} \\
3.56(1.04-5.53)^{\circ}\end{array}$ & $<0.001$ & $<0.001$ & 0.810 \\
\hline $\begin{array}{l}\text { Erythrocyte iron incorporation, \% } \\
\text { IDA } \\
\text { Non-anemic }\end{array}$ & $\begin{array}{c}36.15(26.08-39.35)^{*} \\
16.66(9.33-24.05)\end{array}$ & $\begin{array}{c}33.09(28.84-38.74)^{*} \\
15.89(11.86-24.71)\end{array}$ & $<0.001$ & 0.396 & 0.629 \\
\hline
\end{tabular}

h: hours; n: number; IDA: iron deficient anemia. Medians [interquartile range (IQR)]. * Different between groups $(P<0.001) .{ }^{\circ}$ Within group, different from baseline $(P<0.001)$ Analyzed by linear mixed models with Bonferroni-corrected multiple comparisons.

Table 3. Iron and inflammatory variables 24 hours after vaccination, in the iron deficiency anemia group and the non-anemic group.

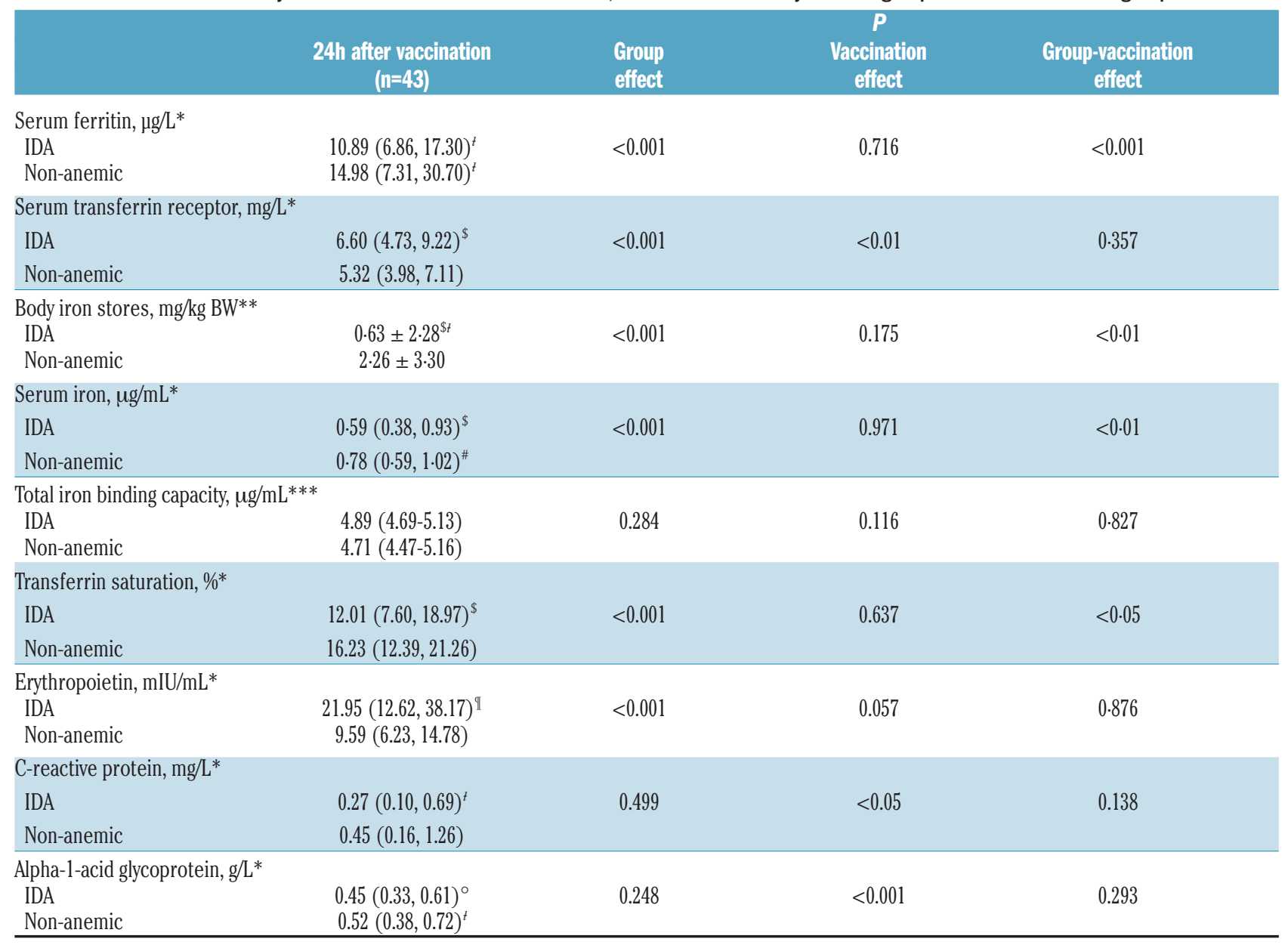

h: hours; n: number; IDA: iron deficient anemia. *Geometric mean [-Standard Deviation (SD), +SD]. **Mean \pm SD. ** Median [interquartile range (IQR)]. ${ }^{*}$ Different between groups $(P<0.001) .{ }^{s}$ Different between groups $(P<0.05)$. ${ }^{\circ}$ Within group, different from baseline $(P<0.001)$ (baseline values shown in Table 1$)$. . Within group, different from baseline $(P<0.01)$ (baseline values shown in Table 1). \#Within group, different from baseline $(P<0.05)$ (baseline values shown in Table 1$)$. Analyzed by linear mixed models with Bonferroni-corrected multiple comparisons. 
tors of SHep were $\mathrm{Hb}(\beta=0.520 ; P<0.01)$ and IL-6 $(\beta=0.508 ; P<0.001)$. In the non-anemic group, at baseline, erythrocyte iron incorporation significantly correlated with SF $(\mathrm{r}=-0.822 ; P<0.001)$, SHep $(\mathrm{r}=-0.792 ; P<0.001)$, $\mathrm{EPO}(\mathrm{r}=0.631 ; P<0.01)$ and $\mathrm{Hb}(\mathrm{r}=-0.475 ; P<0.05)$; at $24 \mathrm{~h}$ after vaccination, incorporation significantly correlated with SHep $(\mathrm{r}=-0.708 ; P<0.001), \mathrm{Hb}(\mathrm{r}=-0.563 ; P<0.01)$ and serum iron $(\mathrm{r}=0.516 ; P<0.05)$. In the IDA group, at baseline and at $24 \mathrm{~h}$ after vaccination, erythrocyte iron incorporation was not significantly correlated with $\mathrm{Hb}$, iron biomarkers, SHep or EPO. In the regression analysis including all subjects, sTfR, Hb, EPO and IL-6 explained 53\% and $50 \%$ of the variation of erythrocyte iron incorporation at baseline and at $24 \mathrm{~h}$ after vaccination, respectively. In all subjects, the only significant predictor of erythrocyte iron incorporation was $\mathrm{Hb}$, both at baseline $(\beta=-0.410 ; P<0.05)$ and $24 \mathrm{~h}$ after vaccination $(\beta=-0.659 ; P<0.01)$. In the regressions including all subjects, SHep, Hb, EPO and IL-6 explained $61 \%$ and $38 \%$ of the variation of serum iron at baseline and at $24 \mathrm{~h}$ after vaccination, respectively. At baseline in all subjects, significant predictors of serum iron were $\mathrm{Hb}(\beta=0.549 ; P<0.01)$ and $\mathrm{EPO}(\beta=-0.333 ; P=0.05)$. In contrast, at $24 \mathrm{~h}$ after vaccination, significant predictors of serum iron were $\mathrm{Hb}(\beta=0.864 ; P<0.01)$, SHep $(\beta=-0.449$; $P<0.05)$ and IL-6 $(\beta=0.347 ; P<0.05)$.

\section{Discussion}

Our main findings are that at $24-36 \mathrm{~h}$ after vaccination: 1 ) there was a comparable 2-3-fold increase in serum IL-6 in both groups $(P<0.001) ; 2)$ there was a significant $>2$-fold increase in SHep in the non-anemic group $(P<0.001)$, but no significant change in SHep in the IDA group; 3$)$ serum iron decreased only in the non-anemic group $(P<0.05)$; and 4$)$ there was no significant change in erythrocyte iron incorporation in either of the two groups; incorporation was approximately 2 -fold higher in the IDA group both before and after vaccination $(P<0.001)$.

Previous experimental human studies have examined the hepcidin response to inflammation. ${ }^{10,11}$ In healthy adults given an infusion of IL-6 (iron status was not reported but subjects were presumably non-anemic), after $2 \mathrm{~h}$ urinary hepcidin increased 7.5-fold, while serum iron and TSAT decreased by $33-34 \% .{ }^{11}$ In healthy adults injected with LPS (iron status was not reported but subjects were presumably
A

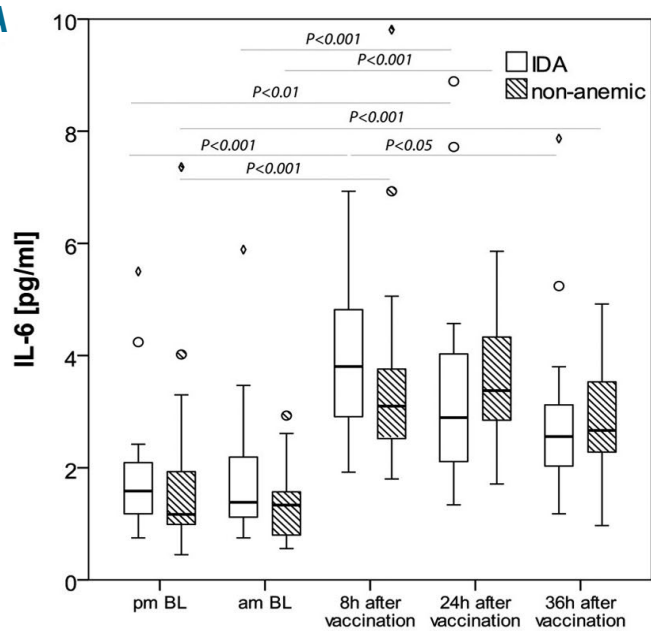

B
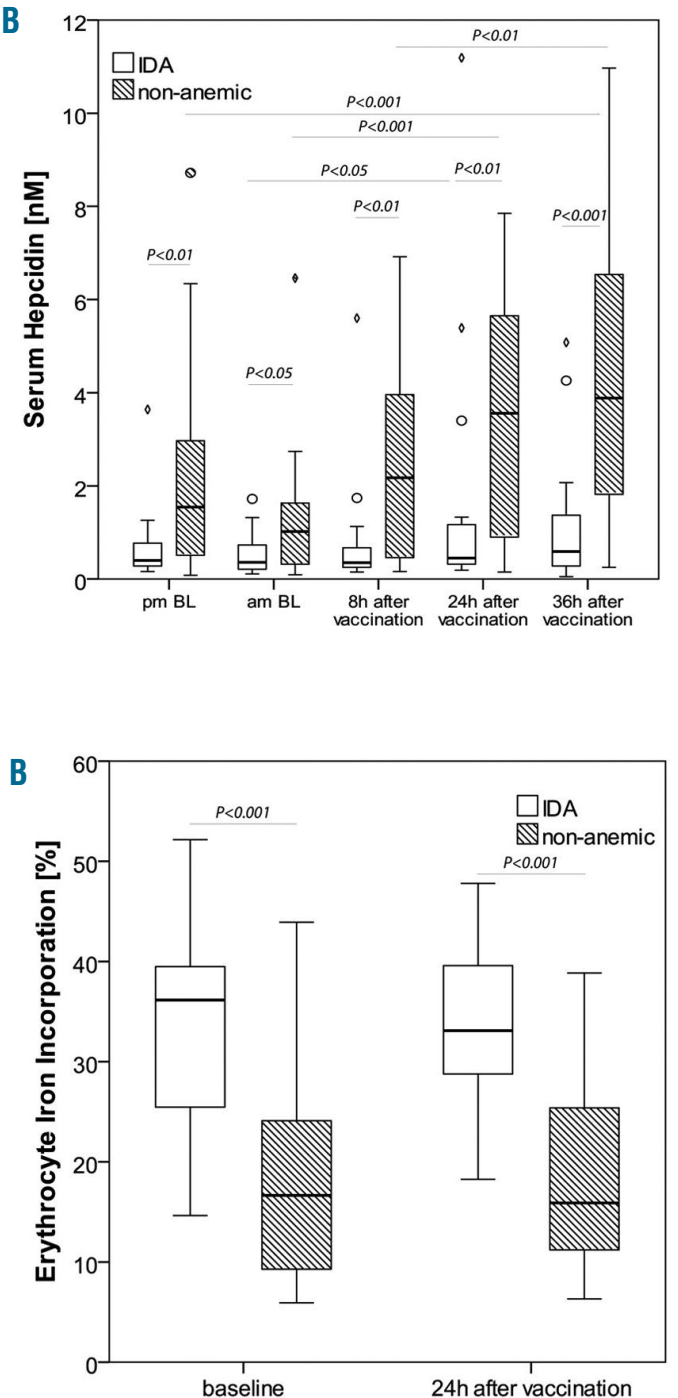

Figure 2. Interleukin- 6 (IL-6) and hepcidin response

influenza/diphtheriatetanus-pertussis (DTP) vaccination. (A) Serum IL-6 and (B) serum hepcidin concentrations before and at 8,24 and 36 hours (h) after vaccination in the iron deficiency anemia (IDA) group $(n=21)$ and the non-anemic group $(n=22)$. BL: baseline.

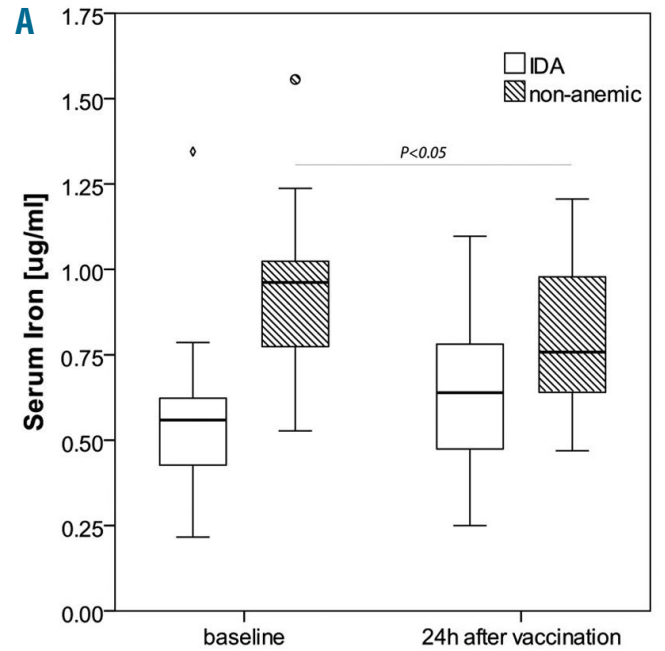

Figure 3. Effects of influenza/diphtheriatetanus-pertussis (DTP) vaccination on serum iron and erythrocyte iron incorporation. (A) Serum iron and (B) erythrocyte iron incorporation (iron absorption and utilization) at baseline $(n=46)$ and at 24 hours (h) after vaccination $(n=43)$ in the iron deficiency anemia (IDA) group and the non-anemic group. 
non-anemic), serum IL- 6 increased within $3 \mathrm{~h}$ after injection, and urinary hepcidin peaked at $6 \mathrm{~h}$, followed by significant hypoferremia. ${ }^{10}$ In a study in Gambian newborns, routine immunizations at birth did not affect serum IL- 6 or SHep at 72-96 $\mathrm{h}$ post vaccination, but the inflammationhepcidin axis was already activated, likely due to the birth process. ${ }^{18}$ However, these studies did not compare responses between anemic and non-anemic subjects or measure effects on iron absorption.

In our study, vaccination induced a rapid and sustained inflammatory response reflected in an approximately 2 - to 3 -fold increase in IL-6 apparent at $8 \mathrm{~h}$ after vaccination and persisting at $36 \mathrm{~h}$ in both the IDA and non-anemic groups (Figure 2A). Despite this, in the women with IDA, SHep did not significantly increase (Figure $2 \mathrm{~A}$ and B). Several factors likely contributed to this effect. High circulating diferric transferrin and high liver iron stores increase hepatic hepcidin synthesis via stimulation of the bone morphogenic protein [sons of mothers against decapentaplegic (BMP$\mathrm{SMAD}$ ) pathway]. ${ }^{1} \mathrm{EPO}$, the main driver of erythropoiesis, stimulates a hepcidin-suppressing factor synthesized in the bone marrow; this factor may be erythroferrone. In a recent study in mice, erythroferrone suppressed hepcidin by inhibiting hepatic BMP/SMAD signaling through BMP5, BMP6, and BMP7. ${ }^{19}$ However, the role of erythroferrone during IDA remains uncertain. ${ }^{20}$ In the IDA group, BMPSMAD signaling was likely suppressed by low TSAT, depleted liver iron stores, and high erythropoietic drive, as indicated by high EPO and sTfR concentrations (Table 1). Our data suggest that moderate IL- 6 stimulation of the Janus kinase/ signal transducer and activator of transcription (JAK/STAT) and BMP-SMAD pathway was unable to overcome this suppression, and SHep remained low. In contrast, in the non-anemic group, BMP-SMAD signaling was not suppressed, and, as a result, IL-6 activation resulted in a rapid increase in SHep. These data suggest that, in mild IDA, low iron status and erythropoietic drive can keep SHep low even in the face of an acute inflammatory stimulus. In addition to the suppression of erythropoiesis by iron restriction through hepcidin, cytokines may directly affect erythropoiesis. ${ }^{21}$ In both the IDA and the non-anemic groups, erythropoiesis appeared to be mildly suppressed 24 $h$ after vaccination, as indicated by a vaccination effect to decrease sTfR and increase EPO (Table 3).

In animal studies, inflammation is a strong inducer of hepcidin, but its effects can be blunted by iron deficiency and/or enhanced erythropoiesis. ${ }^{3-5}$ In mice, erythropoietic drive down-regulated hepcidin even during inflammation induced by LPS injection. ${ }^{3}$ Ferroportin transcription in macrophages may be attenuated by inflammation independent of hepcidin, ${ }^{22,23}$ but activation of Nrf2 reverses this attenuation, ${ }^{24}$ suggesting that iron may dominate over inflammatory stimuli. Conversely, other studies suggest erythroid and inflammatory regulators dominate over iron stores: iron-deficient mice injected with LPS up-regulated hepcidin expression, ${ }^{2}$ while iron-loaded mice with experimentally induced anemia down-regulated hepcidin expression. $^{25}$

Studies in humans with the anemia of chronic inflammation (ACD) and/or IDA suggested erythroid demand for iron is a stronger regulator of hepcidin expression than mild inflammation: SHep was similar between ACD/IDA and IDA, but was higher in ACD; duodenal ferroportin expression was inversely related to SHep and SF, but not to IL-6; and IL-6 levels were similar between ACD (with high
SHep) and ACD/IDA subjects (with low Shep). ${ }^{4}$ In African children, $\mathrm{Hb}$ and SF were positively associated with hepcidin while IL-6 levels were not. ${ }^{26}$ In another study in African children, erythropoietic drive (sTfR) was a much stronger negative predictor of SHep than inflammation. ${ }^{27}$ The differing results of these studies suggest that either inflammation or IDA can be the dominant factor regulating hepcidin, depending on the varying strengths of the opposing stimuli.

In our study, there was a significant and sustained increase in SHep after vaccination in the non-anemic group, with median SHep (nM) more than twice baseline values at 8, 24 and $36 \mathrm{~h}$ after vaccination. We anticipated this would decrease erythrocyte iron incorporation because in our previous studies, acute SHep increases of similar magnitude and duration (approx. 1-2 $\mathrm{nM}$ ) in non-anemic women after the administration of high oral iron doses reduced iron absorption by approximately $40 \% .^{28,29}$ Contrary to our hypothesis, in the present study, there was no significant change in erythrocyte iron incorporation from a labeled test meal given at $24 \mathrm{~h}$ after vaccination, at the peak of the SHep increase, although the women developed mild hypoferremia. Several mechanisms may explain this effect. Hepcidin promotes rapid degradation of ferroportin in liver cells and macrophages reducing iron recycling and serum iron,,$^{30}$ but enterocyte ferroportin may be less sensitive to acute changes in hepcidin. ${ }^{31-34}$ Another potential explanation why high SHep did not effect iron absorption in our study is that inflammation also induced hypoferremia, and the reduction in circulating diferric transferrin was sensed by the enterocyte through TfR1, leading to changes within the enterocyte ${ }^{35,36}$ that induced divalent metal transporter 1 (DMT-1) and ferroportin expression. This may have offset ferroportin degradation by SHep, and allowed continued iron export from the enterocyte. This is supported by our findings that, at $24 \mathrm{~h}$ after vaccination, serum iron was a significant predictor of erythrocyte iron incorporation in the non-anemic group, but not in the IDA group.

The strengths of this study are: 1) we prospectively studied the effects of a standardized (and safe) inflammatory stimulus in both non-anemic women and women with IDA; 2) our subjects were young women who were otherwise healthy and free of potential confounding comorbidities; 3) we precisely quantified erythrocyte iron incorporation (absorption and utilization) using iron stable isotopic labels. Limitation of the study are: 1) we included women in the IDA group who were only mildly anemic, and we induced only a moderate acute inflammatory state. More severe, chronic inflammation and/or anemia may have resulted in differing effects; 2 ) using the stable iron isotope method we measured erythrocyte iron incorporation, which reflects both iron absorption by enterocytes and iron utilization for production of erythrocytes in the bone marrow, and we could not differentiate between these. Finally, we were unable to distinguish whether the lack of SHep increase in response to the inflammatory stimulus in the women with IDA was due to the effect of erythropoietic drive, iron deficiency or both.

To our knowledge, this is the first human experimental study showing that erythrocyte iron incorporation (absorption and utilization of dietary iron) is not reduced in non-anemic subjects by an acute increase in SHep that induces hypoferremia. This finding suggests the enterocyte may be less sensitive to the effect of acute changes in SHep than macrophage iron recycling. This pattern of regulation 
by hepcidin is consistent with the relative contributions of these pathways to the maintenance of body iron homeostasis. Also, to our knowledge, this is the first human experimental study showing that SHep does not increase in anemic subjects after a mild acute inflammatory stimulus. This suggests that, in IDA, iron homeostasis prioritizes correction of iron deficiency, rather than iron withholding, during mild acute infection/inflammation. Our findings provide new insights into how the relative and opposing stimuli that effect hepcidin expression combine to determine net circulating hepcidin, iron absorption and iron homeostasis in young women, and may be clinically rele- vant given that young women are a main target group for iron supplements and fortification to reduce iron deficiency and anemia.

\section{Acknowledgments}

The authors thank all the women who participated in the study, the nursing staff and the other members of the RDCNutrition.

\section{Funding}

They also thank the International Atomic Energy Agency for funding and technical assistance (MOR6022).

\section{References}

1. Sangkhae V, Nemeth E. Regulation of the Iron Homeostatic Hormone Hepcidin. Adv Nutr. 2017;8(1):126-136.

2. Constante M, Jiang W, Wang D, Raymond VA, Bilodeau M, Santos MM. Distinct requirements for Hfe in basal and induced hepcidin levels in iron overload and inflammation. Am J Physiol Gastrointest Liver Physiol. 2006;291(2):G229-237.

3. Huang H, Constante M, Layoun A, Santos MM. Contribution of STAT3 and SMAD4 pathways to the regulation of hepcidin by opposing stimuli. Blood. 2009; 113(15):35933599.

4. Theurl I, Aigner E, Theurl M, et al. Regulation of iron homeostasis in anemia of chronic disease and iron deficiency anemia: diagnostic and therapeutic implications. Blood. 2009;113(21):5277-5286.

5. Darshan D, Frazer DM, Wilkins SJ, Anderson GJ. Severe iron deficiency blunts the response of the iron regulatory gene Hamp and proinflammatory cytokines to lipopolysaccharide. Haematologica. 2010; 95(10):1660-1667.

6. Kearney SL, Nemeth E, Neufeld EJ, Thapa D, Ganz T, Weinstein DA, et al. Urinary hepcidin in congenital chronic anemias. Pediatr Blood Cancer. 2007;48(1):57-63.

7. Gardenghi S, Marongiu MF, Ramos P, et al. Ineffective erythropoiesis in beta-thalassemia is characterized by increased iron absorption mediated by down-regulation of hepcidin and up-regulation of ferroportin. Blood. 2007;109(11):5027-5035.

8. Prentice AM, Doherty CP, Abrams SA, et al. Hepcidin is the major predictor of erythrocyte iron incorporation in anemic African children. Blood. 2012;119(8):1922-1928.

9. Hella J, Cercamondi CI, Mhimbira F, et al. Anemia in tuberculosis cases and household controls from Tanzania: Contribution of disease, coinfections, and the role of hepcidin. PloS One. 2018;13(4):e0195985.

10. Kemna E, Pickkers P, Nemeth E, van der Hoeven H, Swinkels D. Time-course analysis of hepcidin, serum iron, and plasma cytokine levels in humans injected with LPS. Blood. 2005;106(5):1864-1866.

11. Nemeth E, Rivera S, Gabayan V, et al. IL-6 mediates hypoferremia of inflammation by inducing the synthesis of the iron regulatory hormone hepcidin. J Clin Invest. 2004;113(9):1271-1276
12. van der Beek MT, Visser LG, de Maat MPM Yellow fever vaccination as a model to study the response to stimulation of the inflammation system. Vasc Pharmacol. 2002;39(3):117 121.

13. Tsai MY, Hanson NQ, Straka RJ, et al. Effect of influenza vaccine on markers of inflammation and lipid profile. J Lab Clin Med. 2005;145 (6):323-327

14. Hotz K, Krayenbuehl PA, Walczyk T. Mobilization of storage iron is reflected in the iron isotopic composition of blood in humans. J Biol Inorg Chem. 2012;17(2):301-309.

15. Brown E, Hopper J Jr, Hodges JL Jr, Bradley B Wennesland R, Yamauchi H. Red cell, plasma, and blood volume in the healthy women measured by radiochromium cell-labeling and hematocrit. J Clin Invest. 1962;41:2182-2190.

16. Hosain F, Marsaglia G, Noyes W, Finch CA The nature of internal iron exchange in man. Trans Assoc Am Physicians. 1962;75:59-63.

17. WHO. Nutritional anaemias: tool for effective prevention and control. 2017. (Available from: https://www.who.int/nutrition/publications/micronutrients/anaemias-tools-prevention-control/en/Last accessed 8 April 2019.

18. Prentice S, Jallow MW, Prentice AM, Group MR-IN. The effect of BCG on iron metabolism in the early neonatal period: A controlled trial in Gambian neonates. Vaccine. 2015;33(26):2963-2967.

19. Arezes J, Foy N, McHugh $\mathrm{K}$, et al. Erythroferrone inhibits the induction of hepcidin by BMP6. Blood. 2018; 4;132(14):1473 1477

20. Muckenthaler MU, Rivella S, Hentze MW, Galy B. A Red Carpet for Iron Metabolism. Cell. 2017;168(3):344-361.

21. Nemeth E, Ganz T. Anemia of inflammation Hematol Oncol Clin North Am. 2014:28(4):671-681, vi.

22. Guida C, Altamura S, Klein FA, et al. A novel inflammatory pathway mediating rapid hepcidin-independent hypoferremia. Blood 2015;125(14):2265-2275

23. Deschemin JC, Vaulont $S$. Role of Hepcidin in the Setting of Hypoferremia during Acute Inflammation. Am J Hematol. 2013; 88(5):E132-E133.

24. Harada N, Kanayama M, Maruyama A, et al. Nrf2 regulates ferroportin 1-mediated iron efflux and counteracts lipopolysaccharideinduced ferroportin $1 \mathrm{mRNA}$ suppression in macrophages. Arch Biochem Biophys. 2011;508(1):101-109.
25. Nicolas G, Chauvet C, Viatte L, et al. The gene encoding the iron regulatory peptide hepcidin is regulated by anemia, hypoxia, and inflammation. J Clin Invest. 2002; 110(7):1037-1044

26. Cherian S, Forbes DA, Cook AG, et al. An Insight into the Relationships between Hepcidin, Anemia, Infections and Inflammatory Cytokines in Pediatric Refugees: A Cross-Sectional Study. PloS One. 2008;3(12):e4030.

27. Atkinson $\mathrm{SH}$, Armitage AE, Khandwala S, et al. Combinatorial effects of malaria season, iron deficiency, and inflammation determine plasma hepcidin concentration in African children. Blood. 2014; 123(21):3221-3229.

28. Moretti D, Goede JS, Zeder C, et al. Oral iron supplements increase hepcidin and decrease iron absorption from daily or twice-daily doses in iron-depleted young women. Blood 2015;126(17):1981-1989.

29. Stoffel NU, Cercamondi CI, Brittenham G, et al. Iron absorption from oral iron supplements given on consecutive versus alternate days and as single morning doses versus twice-daily split dosing in iron-depleted women: two open-label, randomised controlled trials. Lancet Haematol. 2017; 4(11):e524-e533.

30. Knutson MD, Oukka M, Koss LM, Aydemir F, Wessling-Resnick $M$. Iron release from macrophages after erythrophagocytosis is upregulated by ferroportin 1 overexpression and down-regulated by hepcidin. Proc Natl Acad Sci U S A. 2005;102(5):1324-1328.

31. Laftah AH, Ramesh B, Simpson RJ, et al. Effect of hepcidin on intestinal iron absorption in mice. Blood. 2004;103(10):3940-3944

32. Chaston T, Chung B, Mascarenhas M, et al. Evidence for differential effects of hepcidin in macrophages and intestinal epithelial cells. Gut. 2008;57(3):374-382.

33. Drakesmith $\mathrm{H}, \mathrm{Nemeth} \mathrm{E}, \mathrm{Ganz} \mathrm{T}$. Ironing out Ferroportin. Cell Metab. 2015; 22(5):777-787.

34. Mena NP, Esparza A, Tapia V, Valdes P, Nunez MT. Hepcidin inhibits apical iron uptake in intestinal cells. Am J Physiol Gastrointest Liver Physiol. 2008; 294(1):G192-198.

35. Galy B, Ferring-Appel D, Becker C, et al. Iron regulatory proteins control a mucosal block to intestinal iron absorption. Cell Rep. 2013;3(3):844-857.

36. Taylor M, Qu AJ, Anderson ER, et al Hypoxia-Inducible Factor- 2 alpha Mediates the Adaptive Increase of Intestinal Ferroportin During Iron Deficiency in Mice. Gastroenterology. 2011;140(7):2044-2055 